\section{SOD: STILL A CURE SEEKING A DISEASE}

NEW YORK-Superoxide dismutase (SOD) is a drug searching for a disease. For 25 years, researchers have believed the compound-which reduces the cellular toxicity of superoxides-could rack up huge sales, since such toxicity may lead to degenerative diseases like stroke and cancer, as well as heart and kidney dysfunction. The toxicity could even contribute to aging.

SOD's allure has captivated pharmaceutical majors, as well as biotech companies. U.S. firms currently pursuing SOD include DDI Pharmaceuticals (Mountain View, CA), Biohave believed SOD could rack Technology General (New York), Upjohn (Kalamaup huge sales, since it targets and heart dysfunction. zoo, MI), and Eli Lilly (Indianapolis, IN). European and Japanese companies are also active.

Indeed, SOD market projections are staggering Some reports project SOD drugs for organ transplant cancer, stroke, capturing sales of $\$ 30$ million by 1995 .

Others project sales of $\$ 70$ million by 1995 for cardiovascular uses. These sums relate to first generation SOD drugs, moreover. Market analysts believe subsequent generations could deliver even greater financial returns.

The problems with SOD are many, though. It is unstable and, therefore, difficult to handle. It quickly degrades in the blood stream, often failing to reach the cells it's targeting. It's expensive to make. It causes immune reactions in some patients. And researchers have discovered several forms of the enzyme, leading to speculation that each disease might require a different compound. Finally, efficacy has been nearly impossible to prove.

Though researchers don't know exactly how SOD work, they know it's active during oxidative metabolism. In such metabolism, cells require that oxygen molecules accept four electrons to be completely reduced to two water molecules. This reduction occurs when cytochrome $\mathrm{aa}_{3}$ passes electrons to oxygen. However, oxygen can be partially reduced, resulting in superoxide radicals or hydrogen peroxide. Hydrogen peroxide is toxic to cells. And superoxides attack unsaturated fatty acids and lipids in cellular plasma membranes, destroying cellular integrity. SOD reduces the toxicity of superoxides by converting them to hydrogen peroxide in the presence of two hydrogen ions. The enzyme catalase then converts hydrogen peroxide to water and oxygen. However, researchers believe superoxides are generated faster than SOD can disarm them.

So the 25-year saga of SOD continues. The compound may yet fulfill its promise. Or it could become just another highly touted failure.

-Richard Krebs

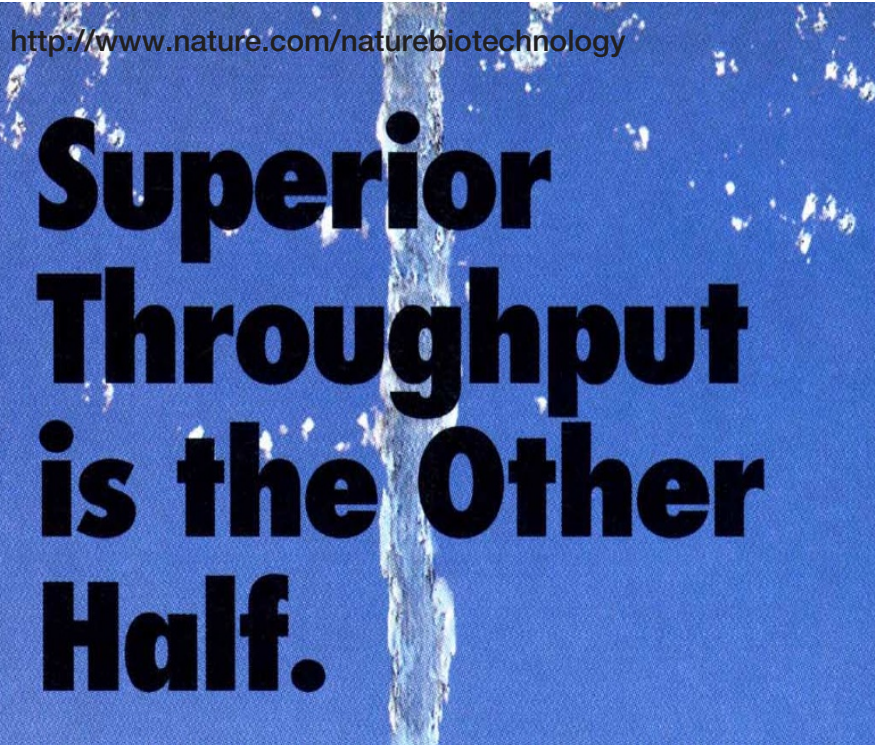

Take the Sartorius Throughput Challenge and see for yourself.

Once again, a side by side comparison proves that not only do our capsule filters flow fasterthey flow longer.

What makes Sartorius capsules so special? Superior throughput performance is achieved via the low protein-adsorption and high void volume of our cellulose acetate membranes. And unparalleled serial filtration is achieved with a double membrane geometry. The first membrane acts as a prefilter, the second, finer pore-sized membrane acts as a final filter.

The results:

Get better product yields

Handle tough-to-filter media with ease

Save time, money and material in the process

So stop settling for less-take the Sartorius

Throughput Challenge and get more: Superior flow rate and better throughput. No other capsule even comes close!

Call (800) 368-7178

Sartorius Corporation

Filtration Products Division 140 Wilbur Place

Bohemia, NY 11716

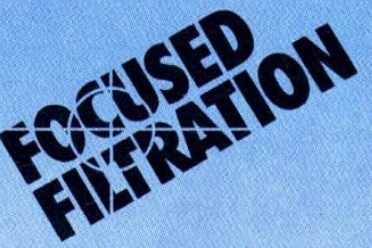

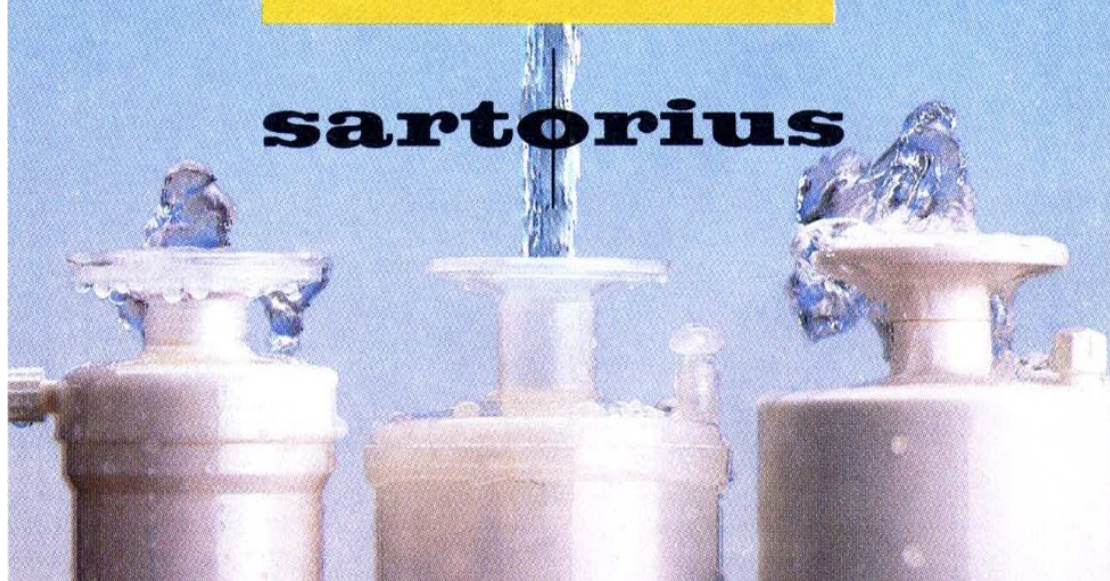

Circle No. 266 on Reader Servi 